\title{
Planung ist gleich Erfolg
}

\begin{abstract}
Was kennzeichnet den Österreichischen Zahnärztekongress 2013 aus Ihrer Sicht?

Das Thema des heurigen Zahnärztekongresses ist entscheidend für den niedergelassenen, praktisch tätigen Zahnarzt, weil nur über eine entsprechende Planung, der eine umfassende Diagnose vorausgeht, eine erfolgreiche Behandlung möglich ist. Aus diesem Grunde haben wir "planungistgleicherfolg“ zum heurigen Generalthema gemacht.

Besonderes Augenmerk haben wir dieses Mal auf die interdisziplinäre Diskussion gelegt, weshalb wir vom Konzept der fachbezogenen Parallelsitzungen abgewichen sind und die Themen in einer Hauptsitzung aufarbeiten.
\end{abstract}

Gibt es Themen, die Ihnen besonders am Herzen liegen?

Das sind besonders Themen, welche die Prävention und die Behandlung älterer Patienten betreffen.

Legen Sie den Fokus mehr auf die wissenschaftlichen Präsentationen, oder haben Informationen für den Praxisalltag auch einen Stellenwert? Ich würde die Begriffe "Wissenschaft" und „Praxisalltag“ ${ }^{\prime \prime}$ keineswegs trennen. Ohne neue wissenschaftliche Erkenntnisse gäbe es keinen Fortschritt in der zahnärztlichen Behandlungspraxis. Natürlich darf uns die Wissenschaft nicht lediglich trockene Zahlen und Fakten fernab jeglicher praktischen Umsetzbarkeit liefern. Wir haben bei der Programmgestaltung besonderes Augenmerk darauf gelegt, dass unsere Kongressgäste, die ja vorwiegend aus der niedergelassenen Zahnärzteschaft kommen, Planungs- und Behandlungsmethoden, die auf dem letzten Stand der wissenschaftlichen Erkenntnisse basieren und die direkt in den Praxisalltag übernommen werden können, angeboten bekommen.

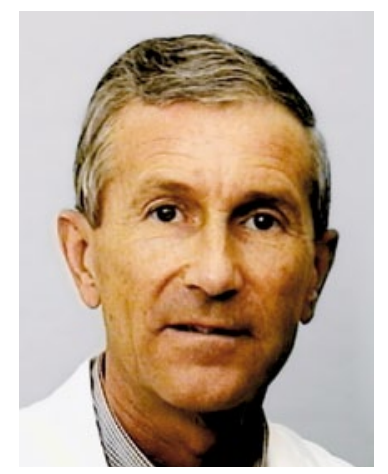

Univ.-Prof. Dr. Rudolf O. Bratschko

Gleichzeitig findet der Jahreskongress des European College of Gerodontology (ECG) statt; gibt es Querverbindungen der beiden Tagungen?

Es freut mich besonders, dass zur selben Zeit der Jahreskongress des European College of Gerodontology in Graz stattfindet, sodass auch die Teilnehmer des österreichischen Zahnärztekongresses das Wissensangebot des ECG nutzen können.

Hat sich die Tätigkeit des Zahnarztes in Österreich in den letzten zehn Jahren gewandelt?

Da unsere Patienten immer älter werden, erhöht sich auch bei uns die Anzahl pflegebedürftiger, aber leider auch dementer Menschen. Wir wissen, dass vor allem in Pflegeeinrichtungen, aber auch bei zu Hause betreuten Personen hier noch ein großer Nachholbedarf besteht. Mobile Zahnarztpraxen für Zahnbehandlungen im Pflegeheim und zu Hause sind technisch längst möglich, werden aber aufgrund der mangelnden wirtschaftlichen Attraktivität kaum durchgeführt. Hier wären die Sozialversicherung gefordert, für Zahnärzte einen entsprechenden Anreiz zu setzen, um solche mobilen Behandlungen anbieten zu können.
Ich hoffe, dass gerade der Kongress für Alterszahnheilkunde auch in diesen Bereichen Lösungsansätze aufzeigen wird.

Hat sich die Ausbildung der Zahnärzte in Österreich zu ihrem Vorteil geändert?

Durch das eigene Studium der Zahnmedizin hat sich das Durchschnittsalter des fertig ausgebildeten Zahnarztes um fast zehn Jahre gesenkt. An der Medizinischen Universität Graz legen wir besonderes Augenmerk auf patientenorientierte, praktische Ausbildung, sodass wir davon ausgehen können, dass unsere Studienabsolventen unmittelbar nach Abschluss des Studiums in der Lage sind, Patienten bestmöglich zu betreuen. Ich sehe darin eine große Verbesserung aus volkswirtschaftlicher Sicht, sowie aus Sicht der Dental Public Health.

Was wünschen Sie sich für die Zukunft der österreichischen Zahnärzte?

Was wünschen Sie Ihren Kongressteilnehmern?

Ihre letzten beiden Fragen erlaube ich mir, aus philosophischer Sicht zu betrachten. Ich wünsche den österreichischen Zahnärzten und unseren Kongressbesuchern die größtmögliche Freude an ihrem Beruf und den größtmöglichen Erfolg bei ihren Zahnbehandlungen, hoffentlich nicht zuletzt aufgrund der beim österreichischen Zahnärztekongress in Graz gewonnenen Erkenntnisse.

Ich freue mich auf ein Wiedersehen beim heurigen Zahnärztekongress.

Danke für das interessante Gespräch!

Dr. Renate Höhl, 2. 8. 2013 